\title{
Solutions of Two-Dimensional Heat Transfer Problems by Using Symmetric Smoothed Particle Hydrodynamics Method
}

\author{
A. Karamanli* and A. Muğan \\ Istanbul Technical University, Faculty of Mechanical Engineering, Gumussuyu 34437, Istanbul, Turkey
}

\begin{abstract}
The symmetric smoothed particle hydrodynamics (SSPH) method is used to generate the basis functions to solve $2 \mathrm{D}$ homogeneous and non-homogeneous steady-state heat transfer problems. The SSPH basis functions together with the collocation method (i.e, the strong formulation of the problem) are used to solve sample problems. Comparisons are made with the results obtained by using different weight functions and particle numbers. The error norms for three sample problems are computed by the use of two different kernel functions such as the revised Gauss function and revised super Gauss function, among which the revised super Gauss function yields the smallest error norm. It is observed that the SSPH method yields large errors for non-homogenous problems, especially if the forcing term is not smooth.
\end{abstract}

\section{Nomenclature}

\begin{tabular}{|c|c|}
\hline $\mathrm{C}(\xi, \mathrm{x}):$ & Symmetric matrice \\
\hline $\mathrm{D}(\xi, \mathrm{x}):$ & A matrice \\
\hline $\mathrm{d}:$ & Radius of the support domain \\
\hline$f:$ & A function \\
\hline$F_{i}:$ & Nodal value of the $j^{\text {th }}$ particle \\
\hline G: & Normalizing constant \\
\hline$g(j):$ & $\mathrm{j}^{\text {th }}$ particle in the compact support of $\mathrm{W}(\xi, \mathrm{x})$ \\
\hline h: & The smoothing length \\
\hline $\mathrm{K}^{(\mathrm{x})}(\xi, \mathrm{x}):$ & A matrice \\
\hline$L_{2}:$ & Global error norm \\
\hline M: & Total number of particles in the problem domain \\
\hline $\mathrm{m}:$ & A number \\
\hline$N(x):$ & $\begin{array}{l}\text { Number of the particles in the compact support } \\
\text { domain }\end{array}$ \\
\hline n: & A number \\
\hline P: & A matrix for Taylor expansion polynomials \\
\hline P: & Source function \\
\hline Q: & A matrix for the unknown variables \\
\hline $\mathrm{T}:$ & Temperature \\
\hline $\mathrm{W}(\xi, \mathrm{x}):$ & Weight function \\
\hline $\mathrm{x}_{\mathrm{i}}, \mathrm{y}_{\mathrm{i}}, \mathrm{z}_{\mathrm{i}}$ : & Physical coordinate direction \\
\hline$\partial:$ & Derivation operator \\
\hline$\sum:$ & Summation symbol \\
\hline$\lambda:$ & The dimensionality of the space \\
\hline$\xi_{\mathrm{i}}:$ & A point in space \\
\hline$\Delta:$ & $\begin{array}{l}\text { The smallest distance between the particle J and its } \\
\text { neighboring particles }\end{array}$ \\
\hline i: & Particle index \\
\hline $\mathrm{j}:$ & Particle index \\
\hline
\end{tabular}

\section{Introduction}

Being the main rival of meshless methods, the finite element method (FEM) is a robust and thoroughly developed method, and it has been widely used in engineering fields due to its versatility for complex geometry and flexibility for many types of linear and nonlinear problems. Most practical engineering problems related to solids and structures are currently solved by using well developed FEM packages that are commercially available. However, the FEM has some inherent shortcomings of numerical methods that rely on the mesh quality and elements. The following limitations of FEM are becoming increasingly evident [1]:

1. High cost in creating an FEM mesh: Creating a mesh for a domain is a prerequisite in using any FEM code. Usually the analyst has to spend most of the time in mesh creation, and it becomes the major component of the cost of a computer aided design (CAD) project especially for three dimensional problems.

2. Low accuracy of stress: Many FEM packages do not predict the stress accurately. The stresses obtained via the FEM are often discontinuous on element boundaries due to the piecewise (or element-wise) continuous nature of the displacement field assumed in the FEM formulation.

3. Difficulty in adaptive analysis: In an adaptive analysis using the FEM, re-meshing (or re-zoning) is required to ensure proper connectivity of elements. To this end, complex, robust and adaptive mesh generation processors have to be developed that are limited to two-dimensional problems. Technical difficulties have precluded the automatic creation of hexahedron meshes for arbitrary three-dimensional domains. In addition, for threedimensional problems, the computational cost of re-meshing at each step is very expensive, even if an adaptive scheme were available. Moreover, an adaptive analysis requires "the mapping" of field variables between meshes in successive stages of the analysis. This mapping process can often lead to additional computation as well as a degradation of accuracy in the solution.

4. Limitation on some analysis types: Under large deformations, considerable loss in accuracy in FEM results can arise from the element distortions. For instance, it is difficult to simulate

*Corresponding author: A. Karamanli, Istanbul Technical University, Faculty of Mechanical Engineering, Gumussuyu 34437, Istanbul, Turkey, Tel: (+90) 53539 92 33, Fax: (+90) 21224951 97, E-mail: armagan_k@yahoo.com

Received May 19, 2012; Accepted June 14, 2012; Published June 17, 2012

Citation: Karamanli A, Muğan A (2012) Solutions of Two-Dimensional Heat Transfer Problems by Using Symmetric Smoothed Particle Hydrodynamics Method. J Appl Computat Math 1:112. doi:10.4172/2168-9679.1000112

Copyright: (c) 2012 Karamanli A, et al. This is an open-access article distributed under the terms of the Creative Commons Attribution License, which permits unrestricted use, distribution, and reproduction in any medium, provided the original author and source are credited. 
crack growth with arbitrary and complex paths which do not coincide with the original element interfaces: In addition, it is very difficult to simulate the breakage of material with large number of fragments and FEM formulations usually lead to a misrepresentation of the breakage path. Serious errors can occur because the problem is non-linear and the results are pathdependent.

The meshless Smoothed Particle Hydrodynamics (SPH) method, proposed by Lucy [2] to study three-dimensional (3D) astrophysics problems, has been successfully applied to analyze solid mechanics and transient fluid mechanics problems. However, it has two shortcomings, namely inaccuracy at particles on the boundary and the tensile instability. Many techniques have been developed to alleviate these two deficiencies, among which are the Corrected Smoothed Particle Method (CSPM) [3,4], the Reproducing Kernel Particle Method (RKPM) [57], the Modified Smoothed Particle Hydrodynamics (MSPH) method [8-11] and the Symmetric Smoothed Particle Hydrodynamics (SSPH) method $[12,13]$. The performance of the CSPH, RKPM, MSPH and SSPH in terms of inaccuracy at particles on the boundary and the tensile instability were already discussed in [3-12]. The MSPH method has been successfully applied to study wave propagation in functionally graded materials, capture the stress field near a crack-tip, and simulate the propagation of multiple cracks in a linear elastic body. The SSPH method is developed to yield symmetric global matrices and has been applied to 2D homogeneous elastic problems successfully.

The SSPH method constructs basis functions that use only locations of particles. These basis functions are found to be similar to those in the Finite Element Methods (FEM) except that the basis for the derivatives of a function need not be obtained by differentiating the function. Needless to say, the basis for the derivatives of a function can be obtained by differentiating the basis function as in the FEM and meshless methods [12].

The SSPH admits a larger class of kernel functions than some other methods such as the SPH, MSPH, RKPM, and the moving least squares (MLS) methods. For finding kernel estimates of derivatives of a function, the SSPH method does not use derivatives of the kernel function while other methods do; instead, the SSPH method uses basis functions different from those employed to approximate the function itself. The kernel function used to generate the basis functions may even be constant.

The SSPH method is applied to some homogeneous solid mechanics problems and proved to be accurate enough to compete with other computational methods. However, originating from its underlying formulations, it has drawbacks for nonhomogeneous problems. Therefore, this study is initiated to reveal the performance of the SSPH method in solving the heat transfer problems, in particular, non-homogeneous problems. It is observed that the SSPH method yields large errors in solving nonhomogeneous problems since it considers nodal values of the forcing term and variation of forcing term in the area among nodes is not considered. Consequently, the error of the SSPH method increases especially if the forcing term is non-smooth. This paper is organized as follows. The SSPH method is described briefly. Then, solutions of different 2D homogeneous and non-homogeneous steady-state heat transfer problems are presented. The conclusions are drawn at the end.

\section{Symmetric Smoothed Particle Hydrodynamics (SSPH)}

The value of a function $f\left(\xi_{1}, \xi_{2}, \xi_{3}\right)$ at a point $\xi=\left(\xi_{1}, \xi_{2}, \xi_{3}\right)$ located in the neighborhood of the point $\mathrm{x}=\left(\mathrm{x}_{1}, \mathrm{x}_{2}, \mathrm{x}_{3}\right)$ can be approximated through the finite Taylor Series expansion provided that continuous derivatives up to the order of $(n+1)$ as follow

$$
f\left(\xi_{1}, \xi_{2}, \xi_{3}\right)=\sum_{m=0}^{n} \frac{1}{m !}\left[\begin{array}{l}
\left(\xi_{1}-x_{1}\right) \frac{\partial}{\partial x_{1}} \\
+\left(\xi_{2}-x_{2}\right) \frac{\partial}{\partial x_{2}}+\left(\xi_{3}-x_{2}\right) \frac{\partial}{\partial x_{3}}
\end{array}\right]^{m} f\left(x_{1}, x_{2}, x_{3}\right)(1)
$$

Neglecting the third and higher order terms and introducing the two matrices $\mathrm{P}(\xi, \mathrm{x})$ and $\mathrm{Q}(\mathrm{x})$, Equation (1) can be written as

$$
\mathrm{f}(\xi)=P(\xi, x) Q(x),
$$

where

$$
\begin{aligned}
& \mathrm{Q}(\mathrm{x})=\left[\begin{array}{l}
f(x), \frac{\partial f(x)}{\partial x_{1}}, \frac{\partial f(x)}{\partial x_{2}}, \frac{\partial f(x)}{\partial x_{3}}, \frac{1}{2} \frac{\partial^{2} f(x)}{\partial x_{1}^{2}}, \\
\frac{1}{2} \frac{\partial^{2} f(x)}{\partial x_{2}^{2}}, \frac{1}{2} \frac{\partial^{2} f(x)}{\partial x_{3}^{2}}, \frac{\partial^{2} f(x)}{\partial x_{1} \partial x_{2}}, \frac{\partial^{2} f(x)}{\partial x_{2} \partial x_{3}}, \frac{\partial^{2} f(x)}{\partial x_{1} \partial x_{3}}
\end{array}\right]^{\mathrm{T}} \\
& \mathrm{P}(\xi-\mathrm{x})=\left[1, \xi_{1}-x_{1}, \xi_{2}-x_{2}, \xi_{3}-x_{3},\left(\xi_{1}-x_{1}\right)^{2}\right. \\
& \left(\xi_{2}-x_{2}\right)^{2},\left(\xi_{3}-x_{3}\right)^{2},\left(\xi_{1}-x_{1}\right)\left(\xi_{2}-x_{2}\right), \\
& {\left[\left(\xi_{2}-x_{2}\right)\left(\xi_{3}-x_{3}\right),\left(\xi_{1}-x_{1}\right)\left(\xi_{3}-x_{3}\right)\right]}
\end{aligned}
$$

The elements of the matrix $\mathrm{Q}(\mathrm{x})$, that are the kernel estimates of a function, its first derivatives and its second derivatives at $\mathrm{x}=\left(\mathrm{x}_{1}, \mathrm{x}_{2}, \mathrm{x}_{3}\right)$, are known variables to be found from Equation (2). If we multiply both sides of Equation (2) with $\mathrm{W}(\xi, \mathbf{x}) \mathbf{P}(\xi, \mathbf{x})^{T}$, we obtain

$$
f(\xi) \mathrm{W}(\xi, \mathrm{x}) \mathrm{P}(\xi, \mathrm{x})^{\mathrm{T}}=\left[\mathrm{P}(\xi, \mathrm{x})^{\mathrm{T}} \mathrm{W}(\xi, \mathrm{x}) \mathrm{P}(\xi, \mathrm{x})\right] \mathrm{Q}(\mathrm{x})
$$

In the compact support of the kernel function $\mathrm{W}(\xi, \mathrm{x})$ associated with the point $\mathrm{x}=\left(\mathrm{x}_{1}, \mathrm{x}_{2}, \mathrm{x}_{3}\right)$, shown in Figure 1, let there be $N(x)$ particles. In the global numbering system, let the particle number of the $j^{\text {th }}$ particle in the compact support of $\mathrm{W}(\xi, \mathrm{x})$ be $\mathrm{g}(j)$. We evaluate Equation (5) at every particle in the compact support of $\mathrm{W}(\xi, \mathrm{x})$ and sum both sides of the equation over these particles to arrive at

$$
\begin{aligned}
& \sum_{j=1}^{N(x)} f\left(\xi^{g(j)}\right) \mathrm{W}\left(\xi^{g(j)}, \mathrm{x}\right) \mathrm{P}\left(\xi^{g(j)}, \mathrm{x}\right)^{T} \\
& =\sum_{j=1}^{N(x)}\left[\mathrm{P}\left(\xi^{g(j)}, \mathrm{x}\right)^{T} \mathrm{~W}\left(\xi^{g(j)}, \mathrm{x}\right) \mathrm{P}\left(\xi^{g(j)}, \mathrm{x}\right)\right] \mathrm{Q}(\mathrm{x})
\end{aligned}
$$

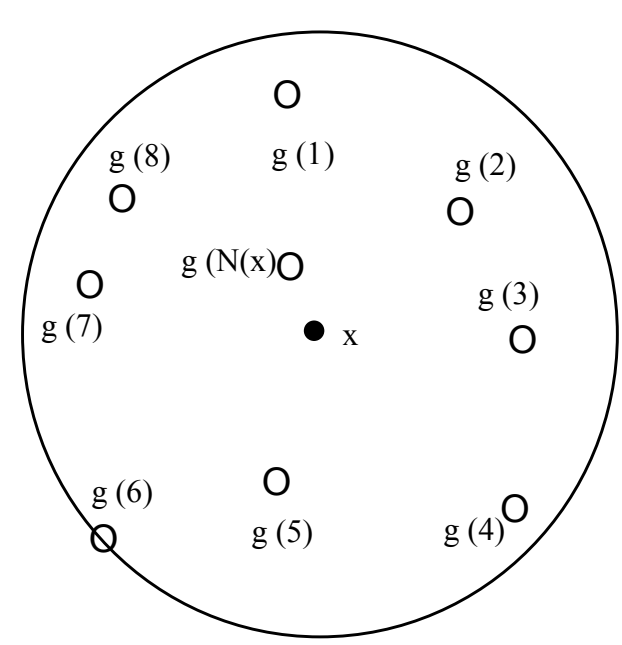

Figure 1: Distribution of particles in the compact support of the kernel function $\mathrm{W}(\xi, \mathrm{x})$ associated with the point $\mathrm{x}=\left(\mathrm{x}_{1}, \mathrm{x}_{2}, \mathrm{x}_{3}\right)[2]$. 
where $\xi^{g(j)}$ denotes the coordinates of the particle $g(j)$. Equation (6) can be rewritten as

$$
\mathrm{C}(\xi, \mathrm{x}) \mathrm{Q}(\mathrm{x})=\mathrm{D}(\xi, \mathrm{x}) \mathrm{F}^{\mathrm{x}}(\xi, \mathrm{x})
$$

where $\mathrm{C}(\xi, \mathrm{x})=\mathrm{P}(\xi, \mathrm{x})^{T} \mathrm{~W}(\xi, \mathrm{x}) \mathrm{P}(\xi, \mathrm{x})$ and $\mathrm{D}(\xi, \mathrm{x})=\mathrm{P}(\xi, \mathrm{x})^{T} \mathrm{~W}(\xi, \mathrm{x})$. It is obvious that the matrix $\mathrm{C}(\xi, \mathrm{x})$ is symmetric; that is why, this technique is called the SSPH method. The set of simultaneous linear algebraic equations in Equation (7) can be solved for the unknown elements of the matrix $\mathrm{Q}(\mathrm{x})$ (Figure 1).

The symmetry of the matrix $\mathrm{C}(\xi, \mathrm{x})$ reduces storage requirements and the CPU time needed to solve Equation (7) for Q(x). None of the matrices in Equation (7) involves derivatives of the kernel function. Thus, a much larger class of functions can be used as the kernel function which improves the practicality and usefulness of the method [13].

For the non-singular matrix $C(\xi, x)$, the solution of Equation (7) is given by

$$
\mathrm{Q}(\mathrm{x})=\mathrm{C}(\xi, \mathrm{x})^{-1} \mathrm{D}(\xi, \mathrm{x}) \mathrm{F}^{\mathrm{x}}(\xi, \mathrm{x})=\mathrm{K}^{(\mathrm{x})}(\xi, \mathrm{x}) \mathrm{F}^{\mathrm{x}}(\xi, \mathrm{x})
$$

where $\mathrm{K}^{(\mathrm{x})}(\xi, \mathrm{x})=\mathrm{C}(\xi, \mathrm{x})^{-1} \mathrm{D}(\xi, \mathrm{x})$. Alternatively, Equation (8) can be written as

$$
Q_{1}(x)=\sum_{j=1}^{M} K_{I J} F_{J}, I=1,2, \ldots 10,
$$

where $F_{I}=f\left(\xi^{\prime}\right)$ and $\mathrm{M}$ is equal to the total number of particles in the entire domain of interest. The value of the function and its derivatives at the point $\mathrm{x}$ are now expressed in terms of the function at all particles in the entire domain [13]. Then, the components of Equation (8) for a 2D problem can be written explicitly as follows

$$
\begin{aligned}
& f(x)=Q_{1}(x)=\sum_{J=1}^{M} K_{1 J} F_{J}, \\
& \frac{\partial f(x)}{\partial x_{1}}=Q_{2}(x)=\sum_{J=1}^{M} K_{2 J} F_{J}, \\
& \frac{\partial^{2} f(x)}{\partial x_{1}^{2}}=2 Q_{5}(x)=\sum_{J=1}^{M} 2 K_{5 J} F_{J}, \\
& \frac{\partial f(x)}{\partial x_{2}}=Q_{3}(x)=\sum_{J=1}^{M} K_{3 J} F_{J}, \\
& \frac{\partial^{2} f(x)}{\partial x_{2}^{2}}=2 Q_{4}(x)=\sum_{J=1}^{M} 2 K_{4 J} F_{J}, \\
& \frac{\partial^{2} f(x)}{\partial x_{1} \partial x_{2}}=Q_{6}(x)=\sum_{J=1}^{M} K_{6 J} F_{J},
\end{aligned}
$$

\section{Numerical Examples}

In this section, three different boundary value problems of steady state heat transfer in rectangular Cartesian coordinates are solved having the following governing differential equation

$$
\frac{\partial^{2} T}{\partial x^{2}}+\frac{\partial^{2} T}{\partial y^{2}}=p(x, y)
$$

where $\mathrm{p}$ is the source function with the boundary condition $T=\bar{T}$ on $\Gamma_{\mathrm{T}}$. The domain of the problem is given in figure 2 . The boundary conditions for all problems in this section are given as the prescribed temperatures at the nodes located on the edges.

Two different weight functions are used to solve 2D homogeneous and non-homogeneous steady-state heat transfer problems. These weight functions are as follows

Revised Super Gauss Function [12]

$$
W(\xi, x)=\frac{G}{(h \sqrt{\pi})^{\lambda}}\left\{\begin{array}{cc}
\left(4-d^{2}\right) e^{-d^{2}} & 0 \leq d \leq 2 \\
0 & d>2
\end{array}\right.
$$

Revised Gauss Function [12]

$$
W(\xi, x)=\frac{G}{(h \sqrt{\pi})^{\lambda}}\left\{\begin{array}{cc}
e^{-d^{2}}-e^{-4} 0 \leq d \leq 2 \\
0 & d>2
\end{array}\right.
$$

where $h$ is the smoothing length, $\lambda$ the dimensionality of the space and G normalizing constant determined by the condition that the integral of the kernel function over the domain is equal to unity. For the circular support domain, the size of the support domain is controlled by the following scaling factor

$$
d=\frac{\sqrt{\left(\xi_{x}^{(i)}-x\right)^{2}+\left(\xi_{y}^{(i)}-y\right)^{2}}}{h}
$$

where $h$ is the smoothing length for particle J which is set equal to $\Delta$ and $\Delta$ is the smallest distance between the particle J and its neighboring particles. 2D homogeneous and non-homogeneous steady-state heat transfer problems are solved for three different uniform particle distributions of $5 \times 10,9 \times 18$ and $17 \times 37$. Convergence of the SSPH method for each problem is calculated by using the following global $\mathrm{L}_{2}$ error norm [14].

$$
L_{2}=\frac{\left\{\sum_{J=1}^{M}\left(T_{\text {num }}{ }^{(j)}-T_{\text {exact }}{ }^{(j)}\right)^{2}\right\}^{1 / 2}}{\left\{\sum_{J=1}^{M}\left\{\left(T_{\text {exact }}^{(j)}\right)^{2}\right\}\right\}^{1 / 2}} \times 100 \%
$$

\section{Example 1}

In this example, we consider that the source function $\mathrm{p}$ is zero. Then, the governing equation and essential boundary conditions are given by

$$
\frac{\partial^{2} T}{\partial x^{2}}+\frac{\partial^{2} T}{\partial y^{2}}=0, T_{1}=T_{2}=T_{3}=0{ }^{\circ} \mathrm{C} \text { and } T_{4}=100{ }^{\circ} \mathrm{C}
$$

that are shown in figure 3.

The analytical solution of this boundary value problem set by Equation (16) can be found as

$$
\frac{T(x, y)}{T_{2}}=\sum_{n=1}^{\infty} \frac{2\left[1-(-1)^{n}\right]}{n \pi} \sin \left(\frac{n x \pi}{a}\right) \frac{\sinh \left(\frac{n y \pi}{a}\right)}{\sinh \left(\frac{n b \pi}{a}\right)}
$$

In numerical solutions, uniformly distributed $5 \times 10,9 \times 18$ and

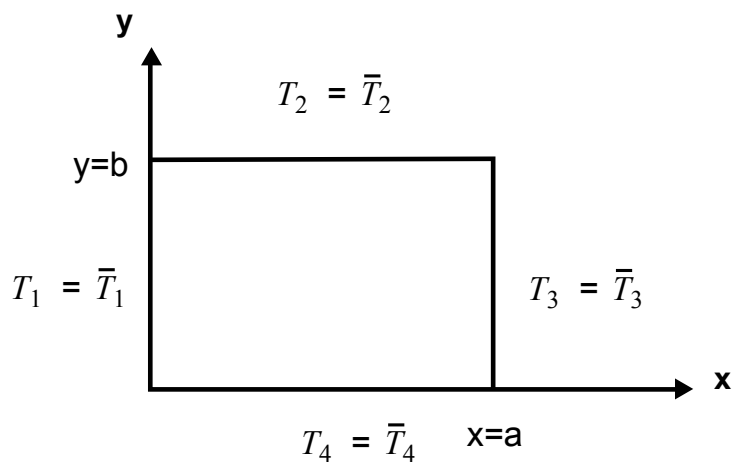

Figure 2: The domain of 2D steady state heat transfer problem. 
17x37 nodes are placed in the problem domain. The smoothing length $\mathrm{h}$ is taken as equal to $\Delta$.

When generating the SSPH basis functions, sufficient number of particles should be included in the kernel function's compact support. For the problems requiring the evaluation of $2^{\text {nd }}$ order derivatives for the collation method, the scaling factor $\mathrm{d}$ should be large enough to have at least six particles in the kernel function's compact support. A larger value of $d$ is not recommended because the CPU time required computing the SSPH basis function increases as $\mathrm{d}$ increases.

Effects of the scaling factor $\mathrm{d}$ on the L2 error norm of temperatures are illustrated in figure 4, where the RGF and RSGF denote Revised Gauss Function and Revised Super Gauss Function, respectively.

It is concluded by examining the curves in figure 4 that the $\mathrm{L}_{2}$ error norms of temperatures decrease as the number of particles increases and the Revised Super Gauss Function always gives the smallest $\mathrm{L}_{2}$ error norm of temperatures. When larger values of $\mathrm{d}$ are used, the $\mathrm{L} 2$ error norm of temperatures for different kernel functions is nearly the same.

\section{Example 2}

In this example, the source function $\mathrm{p}$ is chosen to be non-zero. The governing equation and the essential boundary conditions are given by

$$
\begin{aligned}
& \frac{\partial^{2} T}{\partial x^{2}}+\frac{\partial^{2} T}{\partial y^{2}}=x^{3}+y^{3} \\
& T_{1}=\bar{T}_{2}{ }^{0} C, T_{2}=\bar{T}_{2}{ }^{0} C, T_{3}=\bar{T}_{3}{ }^{0} C, T_{4}=\bar{T}_{4}{ }^{0} C
\end{aligned}
$$

whose particular solution can be found as follows

$$
T(x, y)=\frac{1}{20} x^{5}+\frac{1}{20} y^{5}
$$

In solving this example, uniformly distributed $5 \times 10,9 \times 18$ and $17 \times 37$ nodes are placed in the problem domain. As in Example 1, the smoothing length $\mathrm{h}$ is taken as equal to $\Delta$. The prescribed temperatures on the boundary edges can be found by evaluating Equation (19) on the boundaries. Then, effects of the scaling factor $\mathrm{d}$ on the L2 error norms of temperatures are presented in figure 5 .

It is clear that the $\mathrm{L}_{2}$ error norms of temperatures decrease as the number of particles increases. For the $5 \times 10$ particle distribution, the $\mathrm{L}_{2}$ error norms of temperatures for different kernel functions are almost same. The Revised Super Gauss Function always gives the smallest $\mathrm{L}_{2}$ error norms of temperatures. When the larger value of $\mathrm{d}$ is used, the $\mathrm{L}_{2}$ error norms of temperatures for different kernel functions are nearly the same.

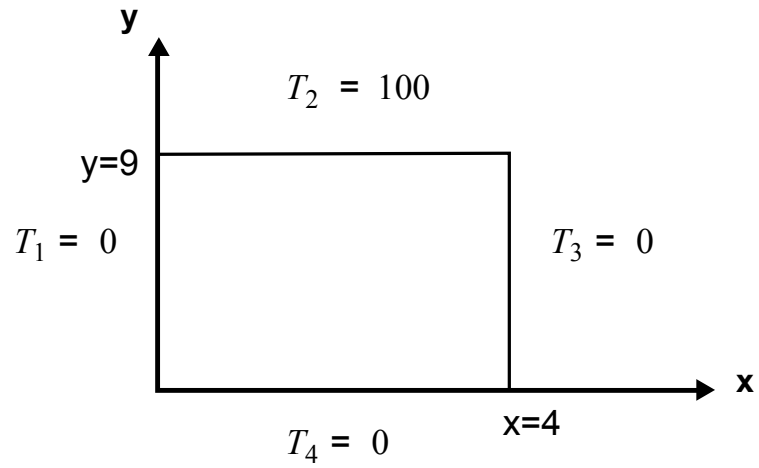

Figure 3: Problem domain and boundary conditions for Example 1.
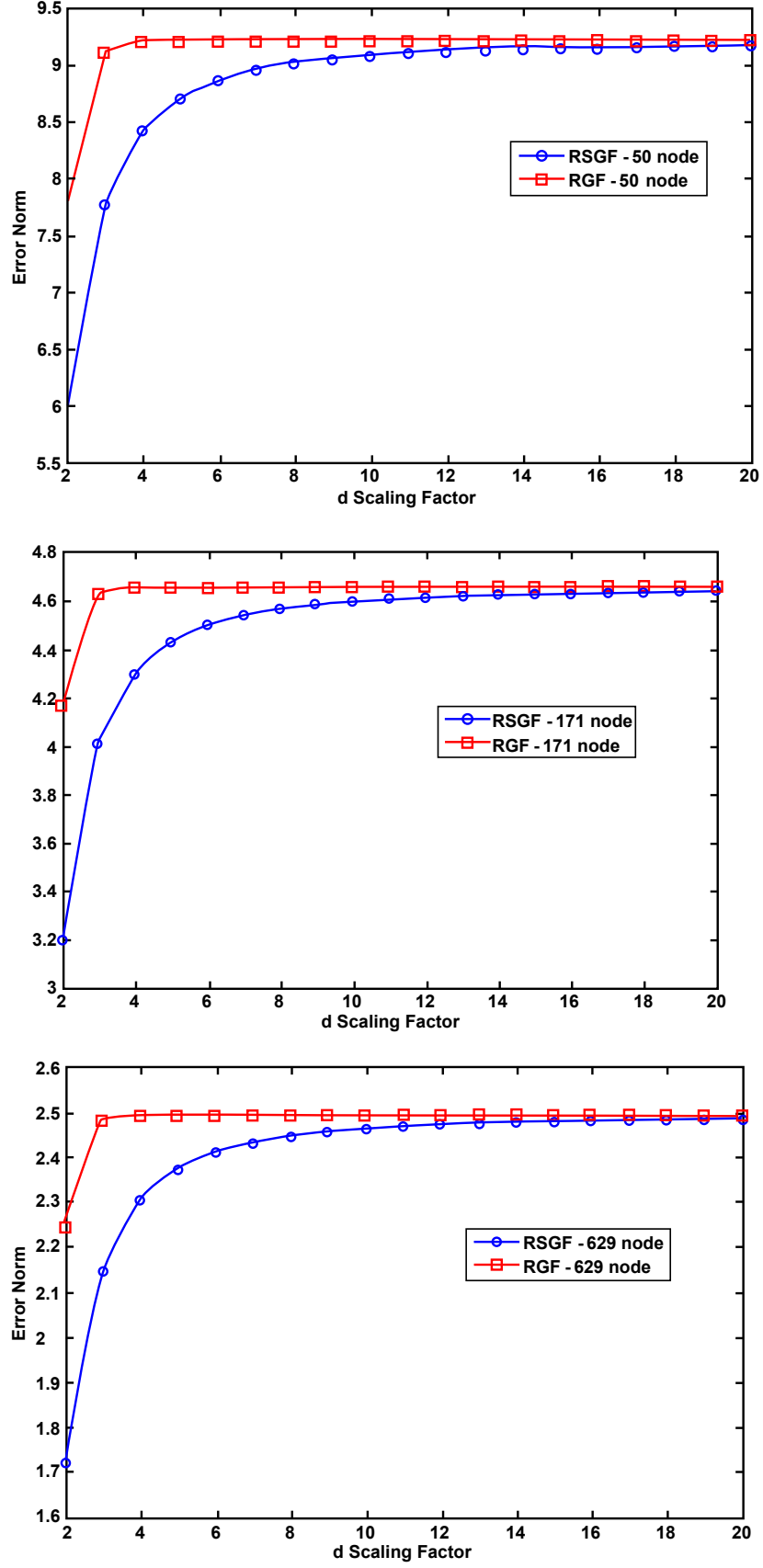

Figure 4: Variation of the $\mathrm{L} 2$ error norms of temperatures as the scaling factor $\mathrm{d}$ changes for different node numbers.

\section{Example 3}

In this example, the source function $p$ is selected as an exponential function. The governing equation and the essential boundary conditions are given by

$$
\begin{aligned}
& \frac{\partial^{2} T}{\partial x^{2}}+\frac{\partial^{2} T}{\partial y^{2}}=e^{x}+e^{y} \\
& T_{1}=\bar{T}_{1}{ }^{0} C, T_{2}=\bar{T}_{2}{ }^{0} C, T_{3}=T_{3}{ }^{0} C, T_{4}=\bar{T}_{4}{ }^{0} C
\end{aligned}
$$

whose particular solution can be found as follows 

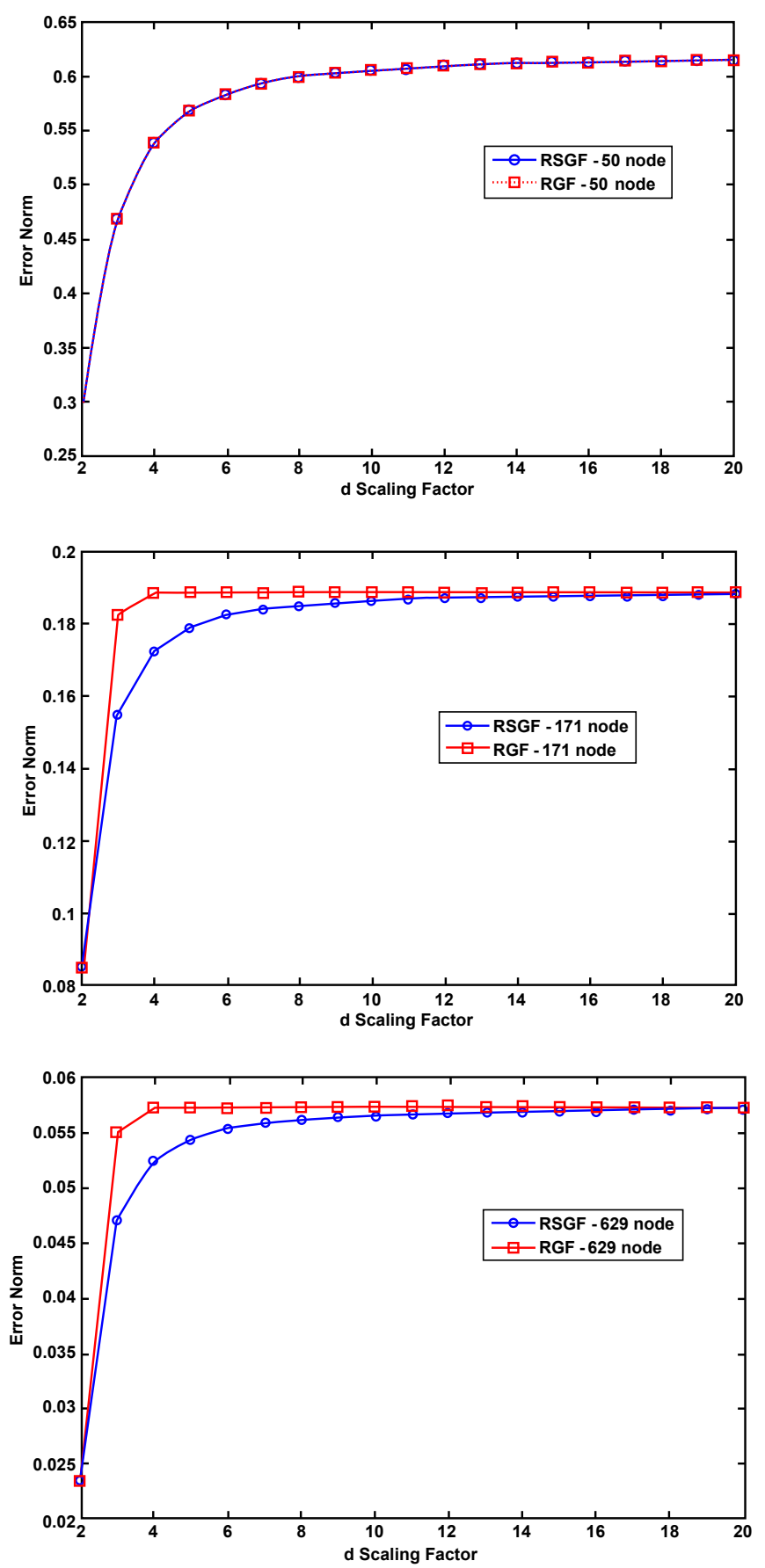

Figure 5: Variation of the L2 error norms of temperatures as the scaling factor d changes for different node numbers.

$$
T(x, y)=e^{x}+e^{y}
$$

Following, uniformly distributed $5 \times 10,9 \times 18$ and $17 \times 37$ nodes are placed in the problem domain. As in the previous examples, the smoothing length $h$ is taken as equal to $\Delta$. The prescribed temperatures on the boundary edges can be found by evaluating the analytical solution given by Equation (11). Effects of the scaling factor $\mathrm{d}$ on the $\mathrm{L}_{2}$ error norms of temperatures are illustrated in figure 6 .

Showing the convergence of the method, the $\mathrm{L}_{2}$ error norms of temperatures decrease as the number of particles increases. For the particle distribution of $5 \times 10$, the $\mathrm{L}_{2}$ error norms of temperatures for different kernel functions are almost the same. The Revised Super Gauss Function always gives the smallest $\mathrm{L}_{2}$ error norms of temperatures. When a larger value of $\mathrm{d}$ is used, the $\mathrm{L}_{2}$ error norms of temperatures for different kernel functions are nearly the same. The convergence of the method by using the Revised Super Gauss Function and Revised Gauss Function is shown in figure 7, where quadratic convergence is observed.
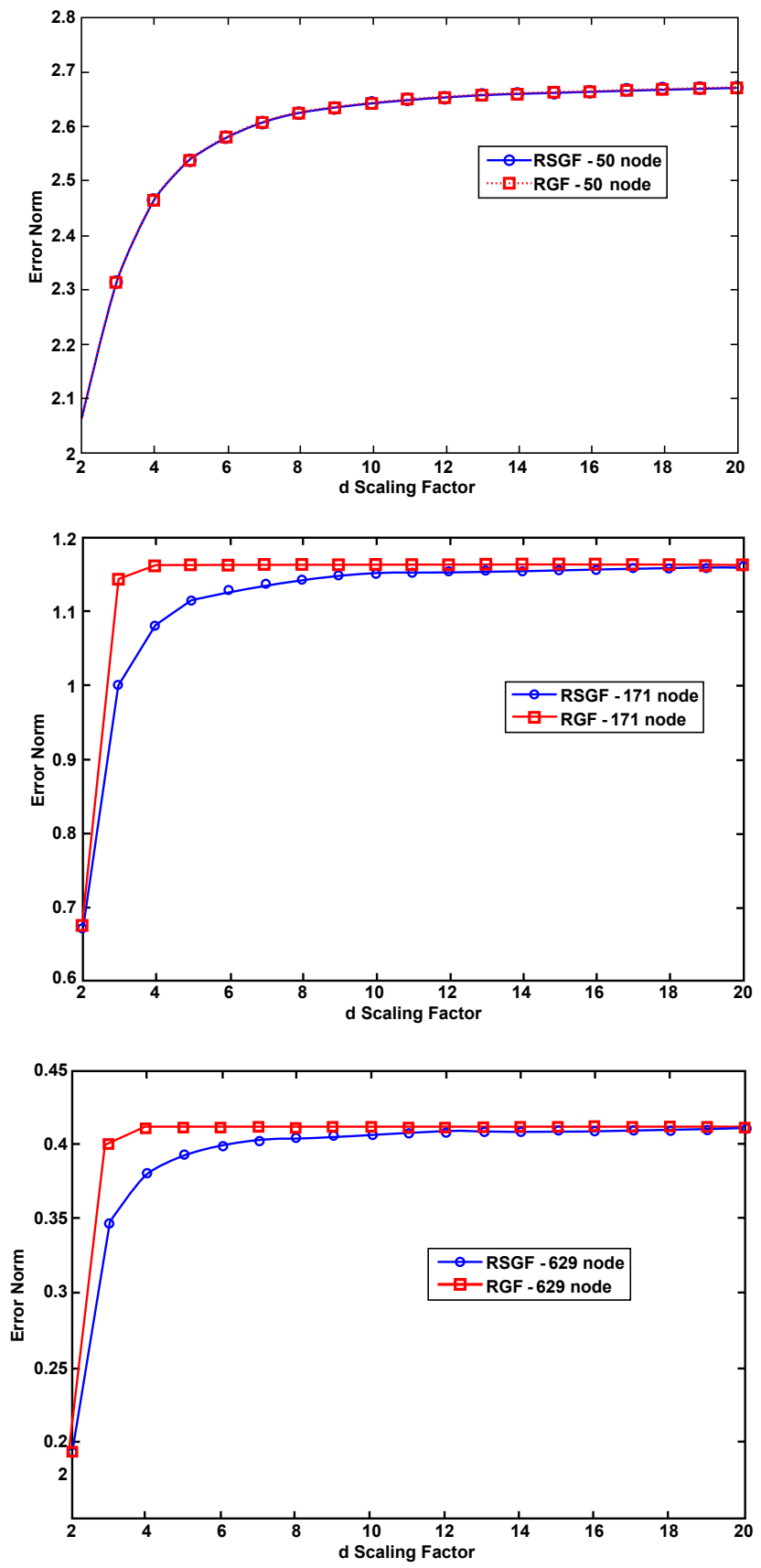

Figure 6: Variation of the L2 error norms of temperatures as the scaling factor $\mathrm{d}$ changes for different node numbers. 


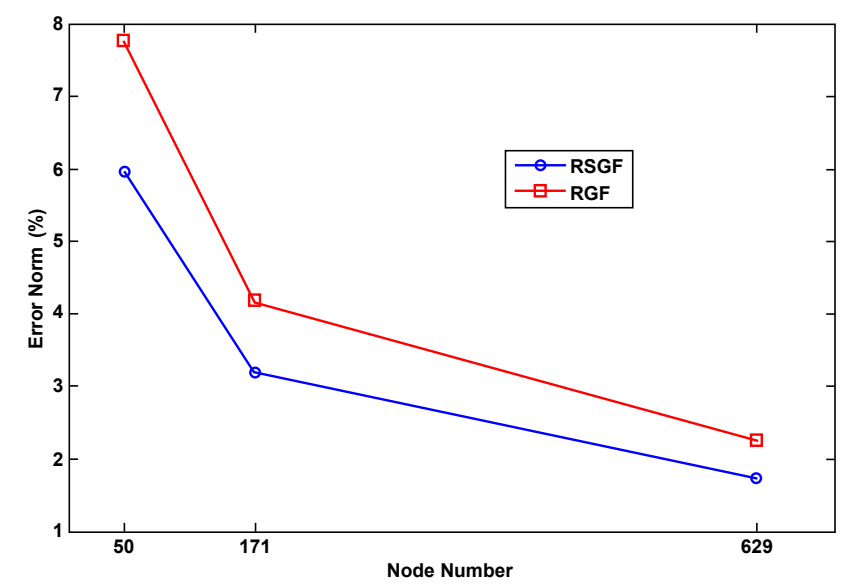

Figure 7: Convergence of the SSPH method by using the weighting functions RGF and RSGF.

\section{Conclusions}

We used the SSPH method to solve 2D homogeneous and nonhomogeneous steady-state heat transfer problems and compared the results obtained by using two different kernel functions and three different particle numbers. The SSPH basis functions are employed to solve three heat transfer problems, two of which are non-homogeneous. According to the numerical results, it is found that the $\mathrm{L}_{2}$ error norm of temperatures decreases as the number of particles increases, that is the evidence of convergence of the method. However, more particles in the kernel compact support require much more CPU times and also numerical errors increase through the large value of the scaling factor d.

It is observed that optimum value of the scaling factor $\mathrm{d}$ to be used for the 2D homogeneous and non-homogeneous steady-state heat transfer problems is 2 and the most suitable kernel function to be used for the SSPH basis function is Revised Super Gauss Function that always yields less error than the Revised Gauss Function. On the other hand, due to the nature of the SSPH formulations that depends on the evaluation of the forcing terms at the nodes, the errors of the SSPH method in nonhomogeneous problems increase regarding to the forcing term, in particular in the existence of non-smooth forcing terms since it does not consider the variation of forcing term in the area among nodes.

Motivated by this fact, future studies will be carried out to develop the performance of the SSPH method in the existence of non-smooth forcing terms, i.e., non-homogeneous problems. Hence, improved accuracy may be obtained for distributed forcing terms.

\section{References}

1. Liu GR, Gu YT (2005) An introduction to meshfree methods and their programming. Springer, Netherlands.

2. Lucy LB (1977) A numerical approach to the testing of the fission hypothesis. Astronomical Journal 82: 1013-1024.

3. Chen JK, Beraun JE, Jih CJ (1999) An improvement for tensile instability in smoothed particle hydrodynamics. Comput Mech 23: 279-287.

4. Chen JK, Beraun JE, Jih CJ (1999) Completeness of corrective smoothed particle method for linear elastodynamics. Comput Mech 24: 273-285.

5. Liu WK, Jun S, Zhang YF (1995) Reproducing kernel particle methods. Int Numer Meth FI 20: 1081-1106.

6. Liu WK, Jun S, Li S, Adee J, Belytschko T (1995) Reproducing kernel particle methods for structural dynamics. Int J Numer Meth Eng 38: 1655-1679.

7. Chen JS, Pan C, Wu CT, Liu WK (1996) Reproducing kernel particle methods for large deformation analysis of non-linear structures. Comput Method App Mech Eng139: 195-227.

8. Zhang GM, Batra RC (2004) Modified smoothed particle hydrodynamic method and its application to transient problems. Comput Mech 34: 137-146.

9. Batra RC, Zhang GM (2004) Analysis of adiabatic shear bands in elastothermo-viscoplastic materials by modified smoothed particle hydrodynamics (MSPH) method. J Comput Phys 201: 172-190.

10. Zhang GM, Batra RC (2007) Wave propagation in functionally graded materials by modified smoothed particle hydrodynamics (MSPH) method. J Comput Phys 222: 374-390.

11. Batra RC, Zhang GM (2007) Search algorithm, and simulation of elastodynamic crack propagation by modified smoothed particle hydrodynamics (MSPH) method. Comput Mech 40: 531-546.

12. Batra RC, Zhang GM (2008) SSPH basis functions for meshless methods, and comparison of solutions with strong and weak formulations. Comput Mech 41 527-545.

13. Zhang GM, Batra RC (2009) Symmetric smoothed particle hydrodynamics (SSPH) method and its application to elastic problems. Comput Mech 43: 321 340.

14. Bodin A, Ma J, Xin XJ, Krishnaswami P (2006) A meshless integral method based on regularized boundary integral equation. Comput Method Appl Mech Eng 195: 6258-6286. 